\title{
Biosorción de Cu(II) sobre cáscaras de maní y maltas residuales. Incorporación de la biomasa contaminada en matrices cerámicas.
}

\section{Biosorption of $\mathrm{Cu}$ (II) ions on peanut shells and brewing waste. Incorporation of contaminated biomass in ceramics.}

Presentación: 6-7/10/2020

\section{Doctorando:}

\section{Cecilia Mazzola}

Grupo de Estudios Ambientales, Facultad Regional San Nicolás, Universidad Tecnológica Nacional, Argentina.

Comisión de Investigaciones Científicas de la provincia de Buenos Aires, Argentina.

cmazzola@frsn.utn.edu.ar

\section{Director/a:}

\section{Nancy Quaranta}

\section{Co-director/a:}

\section{Patricia Blanes}

\section{Resumen}

La presencia de metales pesados en los cursos naturales de agua, tiene efectos nocivos sobre la salud de muchas especies biológicas y sobre el medio ambiente. Los biosorbentes son ampliamente reconocidos como adsorbentes de bajo costo porque son abundantes en la naturaleza o por ser materiales de desecho de la agroindustria. En el presente trabajo se estudia la remoción de iones de $\mathrm{Cu}$ (II) en solución acuosa, utilizando cáscaras de maní y residuos de cebada de la producción de cerveza como materiales adsorbentes. Además se plantea la posibilidad de inmovilizar el metal contaminante junto a la biomasa en matrices cerámicas, comenzando por el estudio de la temperatura óptima de cocción. Se realizó una exhaustiva caracterización de ambas biomasas, y se analizaron numerosas variables en el proceso de biosorción en búsqueda de condiciones que maximicen la capacidad adsorbente de las mismas. Los resultados muestran que las cáscaras de maní logran remociones de hasta el $40 \%$ del metal, mientras que los residuos de cebada no logran superar el 2,3\% de remoción. En base a los resultados obtenidos y dado que ambas biomasas son un subproducto industrial abundante y de bajo costo, se sugiere a la biosorción mediante cáscaras de maní como un proceso efectivo y económico para la remoción de iones de cobre en aguas contaminadas. A su vez, se establecen temperaturas de $950^{\circ} \mathrm{C}-1000^{\circ} \mathrm{C}$ como rango óptimo de cocción de las matrices cerámicas, para analizar la inmovilización de los metales removidos.

Palabras clave: Biosorción, cerámicos, metales. 


\begin{abstract}
The presence of heavy metals in natural water has harmful effects on the health of many biological species and on the environment. Biosorbents are widely recognized as low-cost sorbents because they are abundant in nature, or because they are waste materials from agroindustry. In the present work, the removal of $\mathrm{Cu}$ (II) ions in aqueous solution is studied using peanut shells and brewing wastes as adsorbent materials. In addition, the possibility of immobilizing the metal together with the biomass in ceramic matrices is proposed, starting with the study of the optimal firing temperature. An exhaustive characterization of both biomasses was carried out, and numerous variables in the biosorption process were analyzed in search of conditions that maximize their adsorbent capacity. The results show that peanut shells achieve removals of the metal up to 40\%, while barley residues cannot exceed 2.3\% removal. Based on the results obtained and given that both biomasses are an abundant and low-cost industrial by-products, biosorption using peanut shells is suggested as an effective and economical process for the removal of copper ions in polluted waters. In turn, temperatures of $950^{\circ} \mathrm{C}-1000^{\circ} \mathrm{C}$ are established as the optimum firing range for the ceramic matrices, to analyze the immobilization of the removed metals.
\end{abstract}

Keywords: Biosorption, ceramics, metals.

\title{
Introducción
}

Todos los sectores de nuestra sociedad generan residuos: la industria, la agricultura, la minería, la producción de energía, el transporte, la construcción y los consumidores. Muchos de estos residuos contienen contaminantes, siendo materiales de desecho, materiales de proceso o productos químicos, y son los responsables de la contaminación cuando son liberados en cantidades que exceden la capacidad de asimilación del medio ambiente (Belay, 2010).

Dentro de las clasificaciones de residuos, el término residuo de biomasa se refiere a la materia orgánica originada por un proceso natural o inducido, que queda como material residual del propio proceso productivo, como por ejemplo semillas de girasol, carozos de aceitunas, cáscaras de maní, marlos de maíz, residuos de cebada de la producción de cerveza, etc.

En los últimos años, se ha prestado mucha atención a la eliminación de iones de metales pesados de soluciones acuosas utilizando materiales lignocelulósicos. Los mismos están naturalmente disponibles en abundancia, son baratos, no tóxicos y capaces de adsorber diversos contaminantes a baja concentración. Además, algunos de estos materiales pueden ser regenerados y reutilizados. Estas biomasas tienen varios grupos funcionales tales como hidroxilo, carboxilo, amina, amida, etc., que pueden formar complejos o quelatos con iones de metales pesados, secuestrando fácilmente los mismos (Djemmoe, Njanja, Deussi \& Tonle, 2016).

La biosorción, que puede definirse como la captación de tóxicos mediante el uso de materiales biológicos, se presenta como un procedimiento alternativo a las técnicas de remoción convencionales de metales en solución, como el intercambio iónico o la precipitación química. Este proceso es una tecnología efectiva y de bajo costo si se utiliza el adsorbente adecuado, además de ser muy utilizada y eficaz para el tratamiento de aguas residuales contaminadas por compuestos tóxicos, ya sean orgánicos o inorgánicos (De los Santos, Barajas Fernández, Pérez Hernández, Hernández Rivera \& Díaz Flores, 2019).

En el desarrollo del presente trabajo se estudian diferentes condiciones para la adsorción de iones de Cu (II) sobre cáscaras de maní y residuos de cebada de la industria cervecera.

Una vez adsorbidos, los iones metálicos retenidos tienen que ser eliminados del adsorbente ya sea para su recuperación o para su disposición en forma segura. En la literatura abundan los estudios de adsorción, sin embargo, resulta notoria la escasa 
información científica sobre el tratamiento, el destino final de las biomasas saturadas con el contaminante o bien, la reutilización de estos nuevos residuos de proceso (residuo orgánico + contaminante).

En estas investigaciones se combinan los estudios de biosorción para la remediación de aguas contaminadas con metales pesados, con una vasta experiencia previa del grupo de trabajo respecto a la incorporación de materiales residuales en matrices cerámicas (Quaranta et al., 2011, 2014). Los residuos obtenidos luego de las experiencias de biosorción diseñadas, se utilizarán como materia prima para la producción de materiales cerámicos porosos, determinando especialmente la capacidad de inmovilización de las especies tóxicas estudiadas por parte de las matrices cerámicas, minimizando así las implicancias

ambientales negativas. Esta etapa se comenzó con la determinación de la temperatura óptima de cocción de las piezas cerámicas.

\section{Desarrollo}

Las biomasas en estudio en este trabajo, que se utilizaron como biosorbentes de metales pesados, son cáscaras de maní y residuos de cebada de la industria cervecera. Las primeras, provienen de la etapa de descascarado, que constituye un porcentaje significativo del peso del producto (23\%), las segundas provienen de la etapa de maceración de la producción de cerveza, y se estiman unos $350 \mathrm{gr}$ de residuo húmedo por litro de producto final. Actualmente, ambas biomasas poseen una importante fracción sin una disposición adecuada, constituyendo un material de desecho sin valor de mercado y que puede causar daños ambientales.

Para su uso como adsorbentes, las biomasas se secaron a $100^{\circ} \mathrm{C}$, se molieron y se separaron por tamaño de partícula descartando aquellas mayores a $1000 \mu \mathrm{m}$. Estas biomasas fueron analizadas mediante diversas técnicas para determinar sus características fisicoquímicas y ambientales: microscopía electrónica de barrido (SEM), análisis químico semicuantitativo por espectrometría de dispersión electrónica de rayos X (EDS), difracción de rayos X (XRD), espectroscopia infrarroja de transformada de Fourier (FTIR), pH, determinación de superficie específica BET ( $\mathrm{S}_{\mathrm{BET}}$ ), ecotoxicidad, entre otras.

Las pruebas de adsorción se llevaron a cabo en batch, colocando $100 \mathrm{ml}$ de solución de concentración conocida de $\mathrm{Cu}^{++}$en contacto con las biomasas. La dispersión obtenida se filtró y la concentración residual de $\mathrm{Cu}^{++}$en el filtrado se analizó mediante espectrofotometría visible. Se analizaron diferentes variables del proceso, con el fin de encontrar las condiciones óptimas de adsorción. Entre dichas variables se encuentran: relación adsorbente-adsorbato, tamaño de partículas de la biomasa utilizada y velocidad de agitación.

\section{Resultados}

La Figura 1 muestra las imágenes SEM de las cáscaras de maní y de los residuos de cebada. En ella pueden observarse las estructuras fibrosas de ambas biomasas. En el análisis por EDS se detectaron además de los elementos $\mathrm{C}$ y $\mathrm{O}$ típicos de las estructuras lignocelulósicas, pequeñas cantidades de Al, K y Ca en las cáscaras de maní, y de Mg, Si, P y Ca en la cebada residual.

Las superficies específicas BET determinadas para ambas biomasas resultaron bajas y similares, $0,90 \mathrm{~m}^{2} / \mathrm{g}$ para las cáscaras de maní, y $0,75 \mathrm{~m}^{2} / \mathrm{g}$ para los residuos de cebada. 
Los difractogramas de rayos $\mathrm{X}$ obtenidos mostraron la presencia de los picos característicos de la celulosa semicristalina ( $2 \theta$ : 15,$9 ; 20,9 ; 31,7 ; 44,5$ para cebada y $2 \theta: 16,5 ; 21,9 ; 31,0 ; 44,7$ para maní). Los espectros de FTIR presentaron numerosas bandas que fueron asignadas a las vibraciones de los grupos presentes en los biopolímeros celulosa, hemicelulosa y lignina.
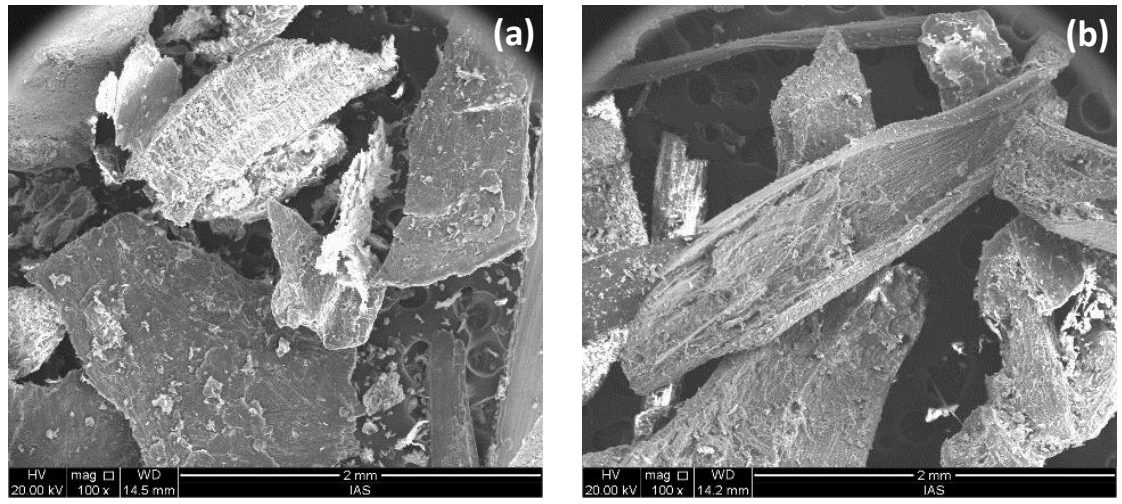

Figura 1. Imágenes SEM de cáscaras de maní (a) y de residuos de cebada (b)

Las experiencias de adsorción llevadas a cabo se analizan teniendo en cuenta las variables estudiadas en cada caso.

Relación adsorbente - adsorbato. La concentración del metal se fijó en $1000 \mathrm{mg} / \mathrm{l}$ y se varió la concentración de las biomasas de 10 a $50 \mathrm{~g} / \mathrm{l}$. Las botellas se agitaron durante 2 horas a $180 \mathrm{rpm}$. Los resultados obtenidos se muestran en la Figura 2, expresados como porcentaje de remoción de cobre total, para ambas biomasas utilizadas como materiales biosorbentes, en función de las concentraciones de biomasa en suspensión.
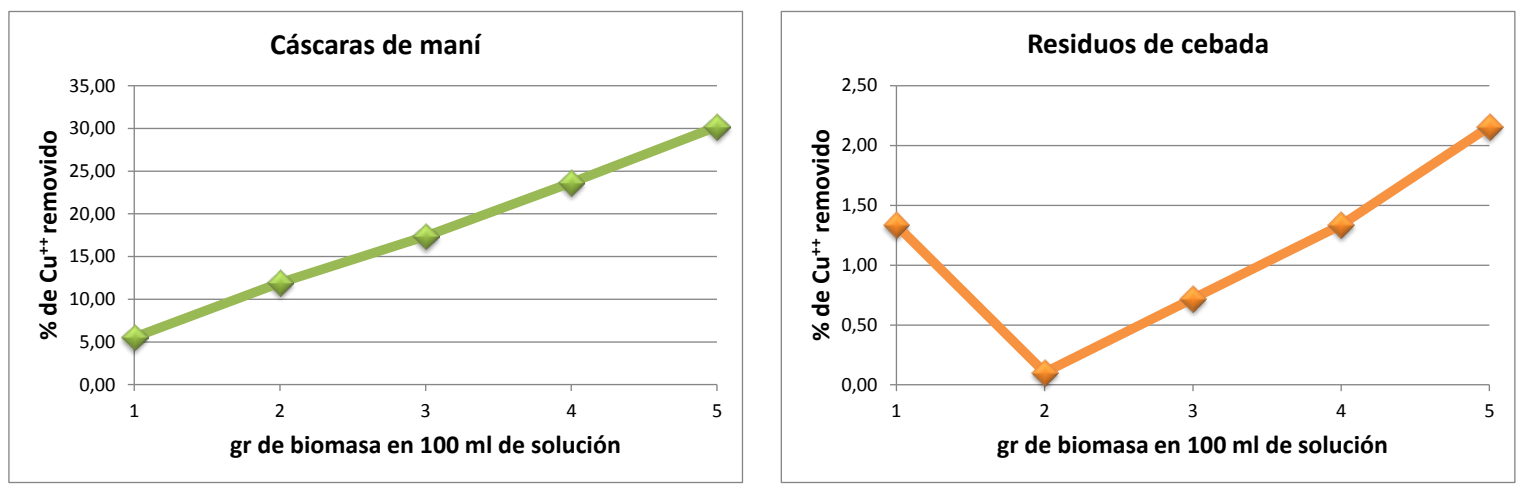

Figura 2. Remoción de $\mathrm{Cu}^{++}$en función de la cantidad de biomasa utilizada

Puede observarse que los mayores porcentajes de remoción de iones metálicos determinados son 30,2\% para las cáscaras de maní y 2,2\% para la cebada residual, ambos para agregados de biomasa de $50 \mathrm{~g} / \mathrm{l}$. Si se evalúan los mg de iones removidos por gr de biomasa, los mejores resultados obtenidos son: $6 \mathrm{mg} \mathrm{de} \mathrm{Cu}^{++} / \mathrm{g}$ de cáscaras de maní (para $50 \mathrm{~g} / \mathrm{l}$ de biomasa) y $1,3 \mathrm{mg}$ de $\mathrm{Cu}^{++} / \mathrm{g}$ de cebada residual (para $10 \mathrm{~g} / \mathrm{l}$ de biomasa).

Tamaño de partículas de la biomasa utilizada. Las biomasas se separaron por tamaño de partículas en 4 porciones diferentes, según se detalla en la Tabla 1. Los ensayos de adsorción se llevaron a cabo en batch, colocando 100 ml de solución de concentración conocida del ión $\mathrm{Cu}^{++}$, en contacto con 5 gr de biomasa y agitando durante 2 horas a 180 rpm. 
Los resultados obtenidos en porcentaje de remoción del cobre fueron de 20,8; 17,6; 41,5; y 33,9 para las cáscaras de maní, y 2,$11 ; 1,01 ; 1,51$ y 2,32 para los residuos de cebada, para los tamaños de partículas A, B, C y D respectivamente. Estos resultados están en relación directa a la $\mathrm{S}_{\mathrm{BET}}$ de las muestras, determinadas para esos rangos de tamaño, datos que se hallan incorporados a la Tabla 1.

\begin{tabular}{|c|c|c|c|}
\hline \multirow{2}{*}{ Referencia } & \multirow{2}{*}{ Tamaño $\mathrm{x}[\mu \mathrm{m}]$} & \multicolumn{2}{|c|}{ Área superficial BET $\left(\mathrm{m}^{2} / \mathrm{g}\right)$} \\
\cline { 3 - 4 } & & Cáscaras de maní & Residuos de cebada \\
\hline A & $\langle 1000($ sin separación) & 0,9027 & 0,7477 \\
\hline B & $1000>\mathrm{x}>250$ & 0,6630 & 0,5394 \\
\hline C & $250>\mathrm{x}>88$ & 1,2333 & 0,6985 \\
\hline D & $88>\mathrm{x}>44$ & 0,9395 & 1,0194 \\
\hline
\end{tabular}

Tabla 1. Tamaños de partículas de las biomasas utilizadas y sus correspondientes áreas superficiales

Velocidad de agitación. Los ensayos se llevaron a cabo con idénticas condiciones a las antes mencionadas, con la biomasa sin separación por tamaño (A), y variando la velocidad del agitador orbital entre 50, 120, 180 y 220 rpm. Los resultados obtenidos, en porcentaje de remoción del cobre, para ambas biomasas, se muestran en la Tabla 2.

\begin{tabular}{|c|c|c|}
\hline \multirow{2}{*}{ Velocidad (rpm) } & \multicolumn{2}{|c|}{$\%$ total de remoción de $\mathrm{Cu}^{++}$} \\
\cline { 2 - 3 } & Cáscaras de maní & Residuos de cebada \\
\hline 50 & 20,69 & 0,43 \\
\hline 120 & 22,50 & 0,50 \\
\hline 180 & 27,83 & 1,80 \\
\hline 220 & 28,38 & 1,92 \\
\hline
\end{tabular}

Tabla 2. Remoción de cobre a diferentes velocidades de agitación

Los resultados muestran nuevamente una capacidad de remoción muy pobre al usar los residuos de cebada de la producción de cerveza artesanal. Para ambas biomasas a partir de $180 \mathrm{rpm}$ la curva de remoción comienza a aplanarse, por lo que esta velocidad se ha tomado como adecuada para los ensayos.

Paralelamente a los ensayos de adsorción, se comenzaron las experiencias de incorporación de las biomasas originales sin contaminar, a las mezclas de arcilla para estudiar los parámetros de producción de las piezas cerámicas. Los ladrillos se conformaron mediante presión uniaxial a $25 \mathrm{MPa}$ en moldes de $70 \mathrm{~mm}$ x $40 \mathrm{~mm}$ y $16 \mathrm{~mm}$ de espesor aproximado. Se confeccionaron muestras con arcilla sola (A) a modo de referencia, y ladrillos con 10\% de agregado en volumen de cáscaras de maní (CM) y de residuos de cebada (RC). En todos los casos se adicionó una humedad del 8\%.

Las piezas en verde producidas se dejaron secar por $24 \mathrm{~h}$ al ambiente, 7 días en estufa a $100^{\circ} \mathrm{C}$, y se sinterizaron a $900^{\circ} \mathrm{C}$, $950^{\circ} \mathrm{C}$ y $1000^{\circ} \mathrm{C}$, siguiendo una rampa de calentamiento de $1^{\circ} \mathrm{C} / \mathrm{min}$, manteniéndose a temperatura máxima durante 3 horas.

Los ladrillos cerámicos obtenidos se han caracterizado por diversas técnicas con el fin de establecer las propiedades de los mismos, en función de las temperaturas de sinterización utilizadas en cada caso. En la Tabla 3 se muestran los resultados obtenidos para: pérdida de peso por calcinación (PPC), variación volumétrica permanente (VVP), módulo de rotura (MOR) resultante de ensayos de flexión y resistencia a la compresión $\left(\sigma_{\mathrm{ROT}}\right)$.

Como puede observarse, para la muestra de referencia A, las mejores propiedades mecánicas se han obtenido a la mayor temperatura ensayada, esto es a $1000^{\circ} \mathrm{C}$. Sin embargo, la VVP se incrementa a más del doble respecto de la cocción a $950^{\circ} \mathrm{C}$. Esto puede generar presencia de microgrietas internas, que con el tiempo perjudiquen la durabilidad del ladrillo cerámico en 
servicio. Por ello, será necesario hacer un estudio microscópico de estos ladrillos antes de establecer la temperatura definitiva a utilizar. Un comportamiento similar se observa para los ladrillos CM y RC, que en general presentan una disminución de las propiedades respecto de la muestra de referencia, pero que cumplen igualmente con los requerimientos del mercado.

\begin{tabular}{|c|c|c|c|c|c|c|c|c|c|}
\hline & A 900 & A 950 & A 1000 & CM 900 & CM 950 & CM 1000 & RC 900 & RC 950 & RC 1000 \\
\hline PPC (\%) & 12,05 & 11,66 & 12,68 & 15,16 & 14,41 & 14,79 & 15,2 & 14,99 & 15,13 \\
\hline VVP (\%) & 5,12 & 6,97 & 15,9 & 6,95 & 8,69 & 15,23 & 4,11 & 7,05 & 14,93 \\
\hline MOR $(\mathrm{MPa})$ & 2,65 & 4,31 & 10,66 & 3,01 & 3,46 & 8,91 & 2,40 & 3,21 & 6,94 \\
\hline$\sigma_{\text {ROT }}(\mathrm{MPa})$ & 23,65 & 25,06 & 101,77 & 17,54 & 20,69 & 48,87 & 16,68 & 18,51 & 52,69 \\
\hline
\end{tabular}

Tabla 3. Principales características de las piezas cerámicas obtenidas

\section{Conclusiones}

Puede concluirse de los estudios realizados hasta el momento, que a pesar de las similares potencialidades de ambas biomasas como materiales adsorbentes, las cáscaras de maní logran, en las condiciones ensayadas, remociones de cobre significativamente mayores que los residuos de cebada. Los resultados de este estudio muestran que las cáscaras de maní pueden ser utilizadas como un material biosorbente efectivo, ecológico y de bajo costo para eliminar los iones $\mathrm{Cu}^{++}$en solución acuosa. En cambio, en iguales condiciones, los residuos de cebada, se comportan como un biosorbente pobre y de baja efectividad.

Los productos cerámicos obtenidos a partir de arcilla, y de mezclas de arcilla y $10 \%$ de biomasa, presentan las mejores propiedades mecánicas a la temperatura de $1000^{\circ} \mathrm{C}$, con valores de módulo de rotura y resistencia a la compresión dentro del rango requerido en el mercado. Sin embargo a esta temperatura, las VVP resultan muy altas, por lo que se requieren estudios microscópicos para determinar la presencia de grietas internas, que podrían afectar el comportamiento en servicio de estos materiales. Por ello, el rango $950^{\circ} \mathrm{C}-1000^{\circ} \mathrm{C}$ se establece por el momento como el adecuado. Con posterioridad a este análisis, una vez determinada la temperatura a utilizar, se evaluará la inmovilización del metal adsorbido sobre dichas biomasas en piezas cerámicas de similares características y en las condiciones experimentales establecidas.

\section{Referencias}

Belay, A.A. (2010). Impacts of chromium from tannery effluent and evaluation of alternative treatment options. Journal of Environmental Protection, 1, 53-58. doi: 10.4236/jep.2010.11007

De los Santos, C.R., Barajas Fernández, J., Pérez Hernández, G., Hernández Rivera, M.A. y Díaz Flores, L.L. (2019). Adsorción de cobre (II) y cadmio (II) en suspensiones acuosas de $\mathrm{CaCO}_{3}$ biogénico nanoestructurado. Boletín de la Sociedad Española de Cerámica y Vidrio, 58(1), 2-13. doi: 10.1016/j.bsecv.2018.05.003

Djemmoe, L.G., Njanja T.E., Deussi M.C.N. y Tonle K.I. (2016). Assessment of copper (II) biosorption from aqueous solution by agricultural and industrial residues. Comptes Rendus Chimie, 19(7), 841-849. doi: 10.1016/j.crci.2016.01.017

Quaranta, N., Caligaris, M., Unsen, M., López, H., Pelozo, G., Pasquini, J. y Vieira, C. (2014). Ceramic Tiles Obtained from Clay Mixtures with the Addition of Diverse Metallurgical Wastes. Journal of Materials Science and Chemical Engineering, 2(2), 1-5. doi: 10.4236/msce.2014.22001

Quaranta, N., Unsen, M., López, H., Giansiracusa, C., Roether, J.A. y Boccaccini, A.R. (2011). Ash from sunflower husk as raw material for ceramic products. Ceramics International, 37(1), 377-385. doi: 10.1016/j.ceramint.2010.09.015 